\title{
H-Mode Power Threshold Studies on MAST ${ }^{\dagger}$
}

\author{
Yasmin Andrew *®D, Jan-Peter Bähner ${ }^{\mathbb{D}}$, Ronan Battle and Tomas Jirman \\ Blackett Laboratory, Imperial College London, Prince Consort Road, London SW7 2BZ, UK \\ * Correspondence: y.andrew@imperial.ac.uk \\ † The MAST Team, United Kingdon Atomic Energy Authority, Culham Centre for Fusion Energy, Abingdon, \\ Oxon OX14 3DB, UK.
}

Received: 30 April 2019; Accepted: 12 July 2019; Published: 19 July 2019

check for updates

\begin{abstract}
Analysis of the L-H and H-L transition power thresholds $\left(P_{t h}\right)$ and pedestal parameters are presented for the mega ampere spherical tokamak (MAST). The dependencies of $P_{t h}$ on the average, core plasma electron density, X-point height, and plasma current are described. Increasing X-point distance from the divertor floor over 10-12 cm is found to increase $P_{t h}$ by a factor of three, while X-point heights greater than this have no further influence. The X-point height dependence of $P_{t h}$ is also observed to be sensitive to the plasma current. An $I_{p}$ decrease from $0.77 \mathrm{MA}$ to $0.65 \mathrm{MA}$, is observed to lower $P_{t h}$ by a factor of three across the X-point height scan and increases the maximum X-point height at which $P_{t h}$ stops increasing by $3 \mathrm{~cm}$. Finally, a comparison of the experimental results with the predictions by the finite beta drift wave model is made, which provides a reasonable condition for the transition into and out of the H-mode.
\end{abstract}

Keywords: L-H transition; H-mode; Tokamak; magnetically confined fusion; pedestal

\section{Introduction}

The high confinement or H-mode [1] is the operational scenario for the next-step device ITER [2]. The edge, radial plasma temperature, and density profiles steepen following the transition from L-mode to H-mode, leading to a reduced core plasma pressure peaking factor, which allows higher stored energy limits $[3,4]$. In addition, the associated steep edge pressure gradients drive a substantial bootstrap current that reduces the need for the current drive [5]. While enhanced stored energy and the bootstrap current are advantages of H-mode, the steep H-mode pressure gradients are also characterized by periodic pedestal collapse known as edge localized modes (ELMs), which release high energy plasma particles to the plasma facing materials.

H-modes are typically accessed when the input heating power crosses a threshold value, leading to a bifurcation in the edge plasma state, with edge or pedestal radial electron density, $n_{e}$, temperature, $T_{e}$, and pressure, $P_{e}$, profiles steepening, and a substantial reduction in the edge plasma turbulence. While the trigger mechanism for the L-H transition has remained elusive, the minimum heating power needed to access the H-mode, the L-H power threshold, $P_{t h}$, has been compared across conventional aspect ratio tokamak devices and the main parametric dependence has been derived to be $[6,7]$ :

$$
P_{t h}=0.0488 \bar{n}_{e}^{0.72} B_{t}^{0.8} S_{A}^{0.94}
$$

where, $\overline{n_{e}}$ is the plasma line average density $\left(\times 10^{20} \mathrm{~m}^{-3}\right), B_{t}$ is the toroidal magnetic field at the magnetic axis $(\mathrm{T})$, and $S_{A}$ is the plasma boundary surface area $\left(\mathrm{m}^{2}\right)$.

In addition to these global parameters, it is known that each device H-mode access $P_{t h}$ has other dependences, which are referred to as "hidden variables". These include variations in the plasma boundary shape (number of $X$-points, magnetic balance, radial, poloidal, vertical location of the X-points, and plasma elongation), plasma ion species, applied 3D non-axisymmetric fields, wall conditioning 
techniques, and neutrals. The dependences of the $\mathrm{L}-\mathrm{H}$ and $\mathrm{H}-\mathrm{L}$ transition $P_{t h}$ on these hidden variables are receiving renewed interest because of the anticipated heating power availability on ITER, and the requirement to access and remain in the H-mode early in ITER operation in hydrogen or helium plasmas, prior to high activation phases with deuterium and tritium $[7,8]$.

Motivated by the need to further understand and control H-mode access on future tokamaks and in preparation for upcoming experiments on MAST- $\mathrm{U}$, the $P_{t h}$ for the $\mathrm{L}-\mathrm{H}$ and $\mathrm{H}-\mathrm{L}$ transitions dependence of MAST on the X-point height has been analyzed and is presented here. A wider dataset of plasmas has been included in this study to explore the X-point height operation space in more detail than has been presented in previous papers on this topic. The paper is structured as follows: Section 2 describes the experimental set-up, the data analysis for the $P_{t h}$ and pedestal parameter dependence on $\left\langle\bar{n}_{e}\right\rangle$; the results from a study on the effect of X-point height on $P_{t h}$ are presented in Section 3 and a comparison of the pedestal parameters is made the finite beta drift wave model [9] for zonal flow suppression of turbulence at the L-H transition in Section 4. Finally, the main conclusions from the study are summarized in Section 5.

\section{Density Dependence}

The L-H and H-L transition times have been identified for a series of plasmas to study MAST $\mathrm{H}$-mode access and exit dependencies on core plasma $n_{\mathrm{e}}$ on MAST which had a major radius, $R=0.85 \mathrm{~m}$ and minor radius, $a=0.65 \mathrm{~m}$. These shots were run with high field side (HFS) deuterium fueling in the connected double null diverted (CDND) magnetic configuration, an example of which is shown in Figure 1, in which most MAST H-modes were accessed [10-13]. The L-mode target density was controlled using active feedback, while neutral beam injection was constant in each shot and varied from shot to shot by a minimum of $0.2 \mathrm{MW}$. It is important to note that a minimum density for accessing H-mode on MAST was typically set by the occurrence of low-density locked modes (in the region of $n_{e}=1.5 \times 10^{19} \mathrm{~m}^{-3}$, at plasma current of $I_{p}=0.65 \mathrm{MA}$ and at increasing $n_{e}$ with $I_{p}$.) The results presented in this paper were above the locked mode density minimum.

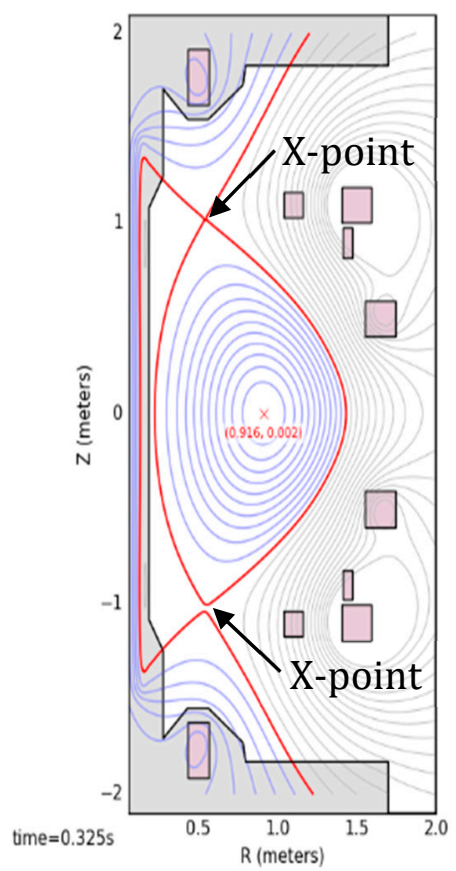

Figure 1. MAST's connected double null magnetic configuration. 
All shots included for the density scan study had Ip/Bt values of $0.70 \mathrm{MA} / 0.45 \mathrm{~T}$. The threshold power for $\mathrm{H}$-mode access and exit is defined in this study as:

$$
P_{\text {th }}=P_{\text {in }}+P_{\mathrm{OH}}-\frac{d W}{d t}
$$

at the $\mathrm{L}-\mathrm{H}$ and $\mathrm{H}-\mathrm{L}$ transitions, where $P_{\text {in }}$ is the total additional (NBI) heating power, $P_{\mathrm{OH}}$ is the ohmic power, and $d W / d t$ is the rate of change of the stored plasma energy. In the absence of the availability of power ramps for these shots, the fluctuations in the plasma density and net input power, defined in Equation (2), were taken to provide sufficient variation in plasma parameters to cause the transitions and provide a measurement of the power threshold at the forward and back H-mode transitions and the associated pedestal parameters.

The general plasma parameters for a typical shot with a forward and back transition is shown in Figure 2. The $\mathrm{L}-\mathrm{H}$ transition is taken as the point at which a sharp drop in the divertor $\mathrm{D}_{\alpha}$ signal occurs along with a sharp rise in the core $n_{e}$ and the stored plasma energy, W. Many of these $\mathrm{L}-\mathrm{H}$ transitions are preceded by a dithering or intermediate phase, in which some oscillations in the divertor $D_{\alpha}$ signal occurs or possibly very high frequency, very small ELMs, as indicated in Figure 2. The transition into these intermediate phases have not been considered in the analysis carried out for this study. The transitions out of the $\mathrm{H}$-mode have similar signal signatures but in reverse, many $\mathrm{H}-\mathrm{L}$ transitions are also triggered by large ELMs or MHD events, from which the confinement does not subsequently recover.

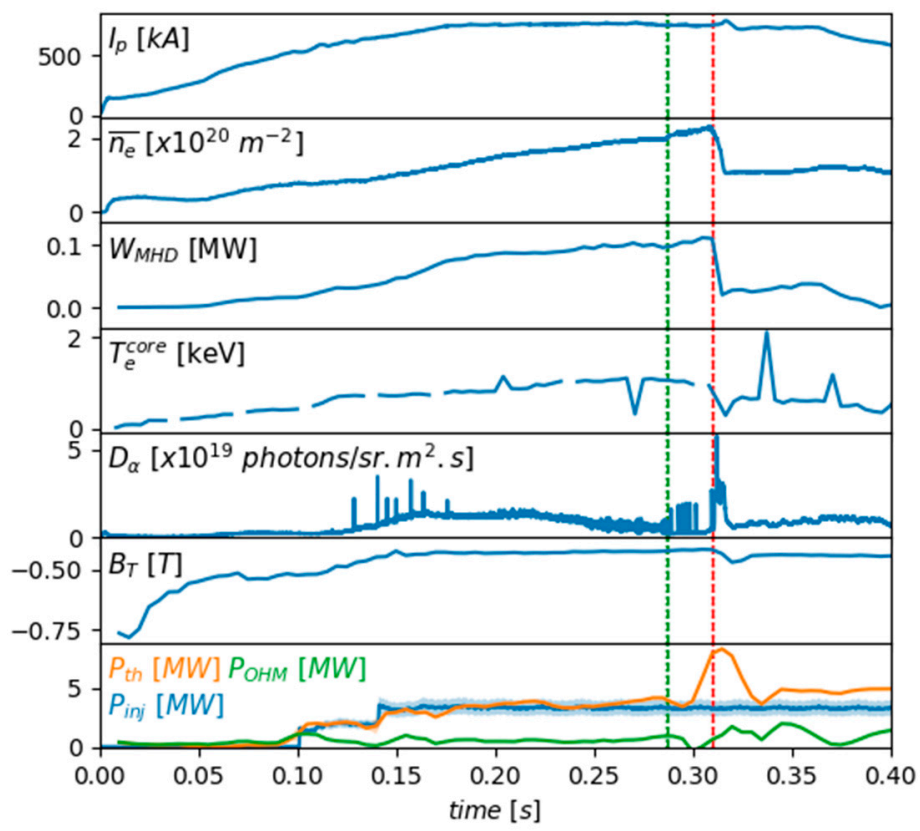

Figure 2. General plasma parameters for a shot 27035 from the density/X-point height scan at $0.7 \mathrm{MA} / 0.43 \mathrm{~T}$.

The pedestal electron density, $n_{e}^{\text {ped }}$, and temperature, $T_{e}^{\text {ped }}$, in these shots has been measured with a multi-radial point, multi-time edge Thomson scattering diagnostic with a radial resolution of $\Delta R=10 \mathrm{~mm}$ [14-16]. Example low field side L-mode and H-mode Thomson scattering diagnostic measured $n_{e}$ profiles are shown in Figure 3a. A modified hyperbolic tangent function, which includes an additional quadratic term, has been used to fit the pedestal data [17], as shown in Figure 3a,b. These fits to the pedestal data have been to identify the parameters and regions of interest in the edge plasma. A weak density pedestal often develops prior to the $\mathrm{L}-\mathrm{H}$ transition, both in the absence and presence of the dithering or intermediate phases. These $n_{e}$ profiles have been used to identify 
the pedestal top or "knee" and the region of steepest density gradient, as close to and prior to the L-H and $\mathrm{H}-\mathrm{L}$ transitions. The corresponding value of $T_{e}$ at these radial locations (the knee and the steepest $n_{e}$ gradient) have then been used in the subsequent analysis. For the back transitions, the pedestal values have been taken in $\mathrm{H}$-mode, as close to the transition as possible. The $\mathrm{H}-\mathrm{L}$ parameters are therefore more easily identifiable and have smaller fitting errors due to the often much stronger pedestal shape in the H-mode, as shown in Figure 3a.

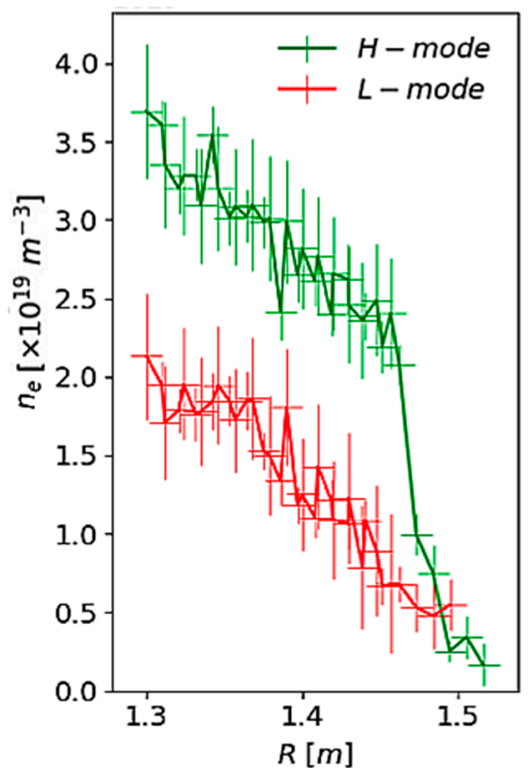

(a)

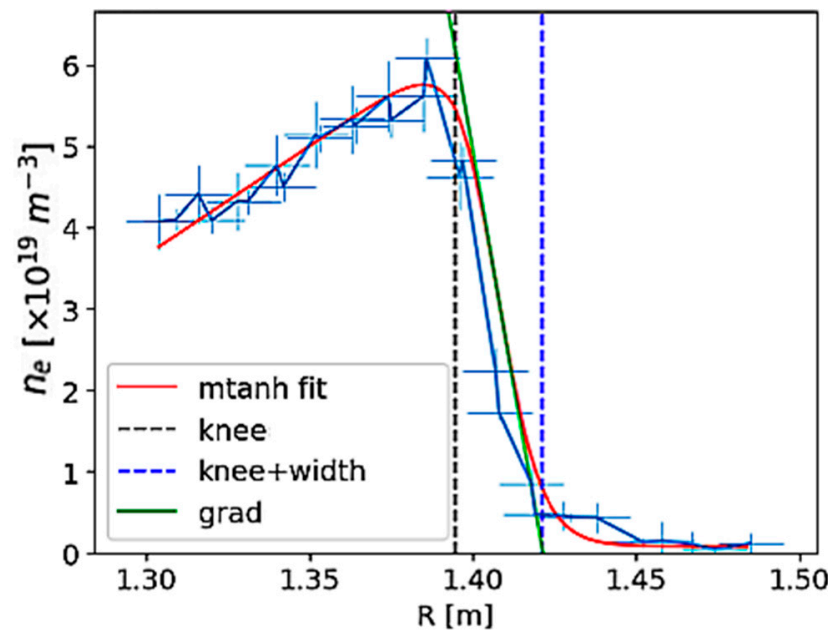

(b)

Figure 3. TS measurements on the pedestal $n_{e}$ for (a) typical L-mode and H-mode profiles for shot number 27035 and (b) with the modified tanh fit to the with the relevant pedestal parameters labeled, knee, width, and steepest $n_{e}$ gradient.

The values of the power threshold, $P_{t h}$, for the L-H and H-L transitions, $P_{L H}$ and $P_{H L}$, are shown in Figure $4 \mathrm{a}$ as a function of averaged core $\overline{n_{e}}$ along with pedestal values in Figure $4 \mathrm{~b}$ of $n_{e}^{\text {ped }}, T_{e}^{\text {ped }}$, and $P_{e}^{p e d}$, taken at the pedestal knee, over a similar density range. The $P_{L H}$ shows an increase of a factor of two over the density range of $1-4 \times 10^{19} \mathrm{~m}^{-3}$, while the smaller $P_{H L}$ dataset indicates a weaker density dependence. Fits to these data provide the following dependences on the core density:

$$
\begin{aligned}
& P_{L H} \propto{\overline{n_{e}}}^{0.77( \pm 0.01)} \\
& P_{H L} \propto{\overline{n_{e}}}^{0.54( \pm 0.07)}
\end{aligned}
$$

Due to the limited amount of data available for the $\mathrm{L}-\mathrm{H}$ transition, a linear $P_{L H}$ dependence on $\overline{n_{e}}$ is used for the rest of the analysis presented in this paper. It is interesting to note that there is no indication of a low-density turning point in either $\mathrm{P}_{\mathrm{LH}}$ or $\mathrm{P}_{\mathrm{HL}}$ over this density range, indicating that it lies in the high-density, linear branch of $\mathrm{H}$-mode access.

Despite the increase in the threshold power, for both $\mathrm{L}-\mathrm{H}$ and $\mathrm{H}-\mathrm{L}$ transitions, from 1.8 MW to $4 \mathrm{MW}$ with the increase in core $\overline{n_{e}}$ and $n_{e}^{\text {ped }}$, the pedestal temperature, $T_{e}^{\text {ped }}$, shows very little variation with values at around $100 \mathrm{eV}$ across the density range, as shown in Figure $4 \mathrm{~b}$. The dependence of the pedestal pressure, $P_{e}^{\text {ped }}$, on the core $\overline{n_{e}}$ is dominated by the linear dependence on $n_{e}^{\text {ped }}$. These results are interpreted as a further indication that a threshold in edge region $T_{e}$ or a related parameter such as the pedestal ion temperature, $T_{i}$, or the radial electric field, $E_{r}$, is important for H-mode access [18-20]. The very weak dependence of $T_{e}^{\text {ped }}$ on both core and pedestal $n_{e}$ also confirms that the density range considered is in the high density, linear H-mode branch $[19,21,22]$. In the absence of spatially resolved 
pedestal ion temperature and rotation velocity measurements, it is not possible to comment further on whether the pedestal electron or ion channel is the dominant player in the $\mathrm{L}-\mathrm{H}$ and $\mathrm{H}-\mathrm{L}$ transition.

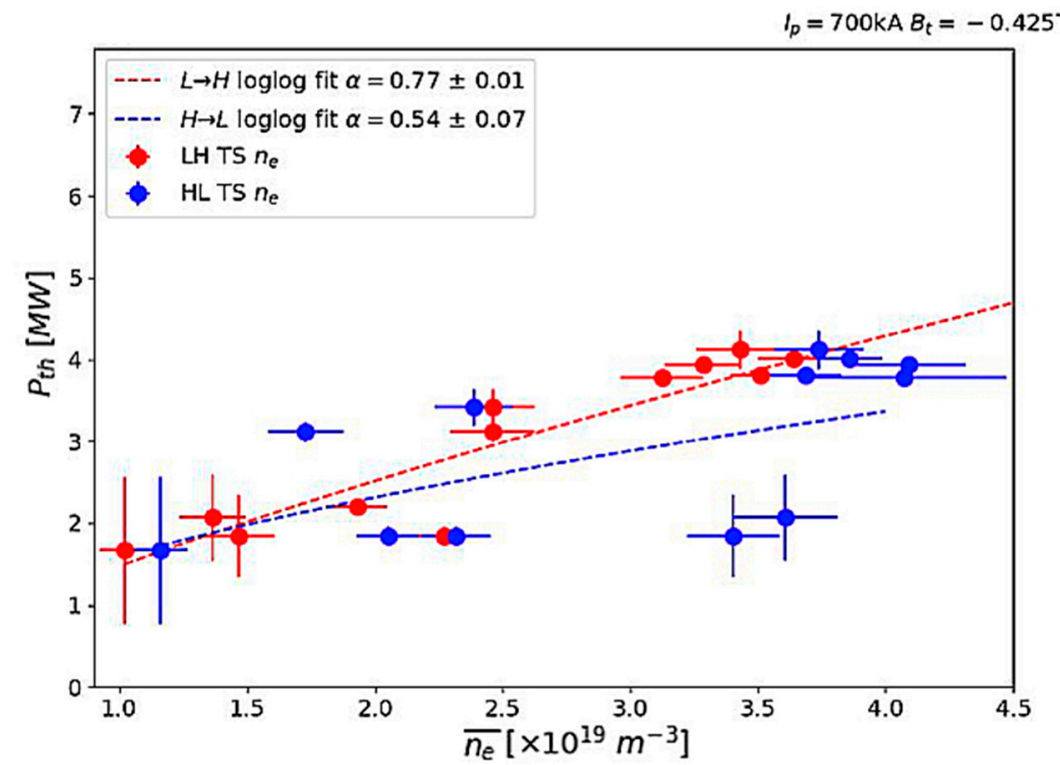

(a)
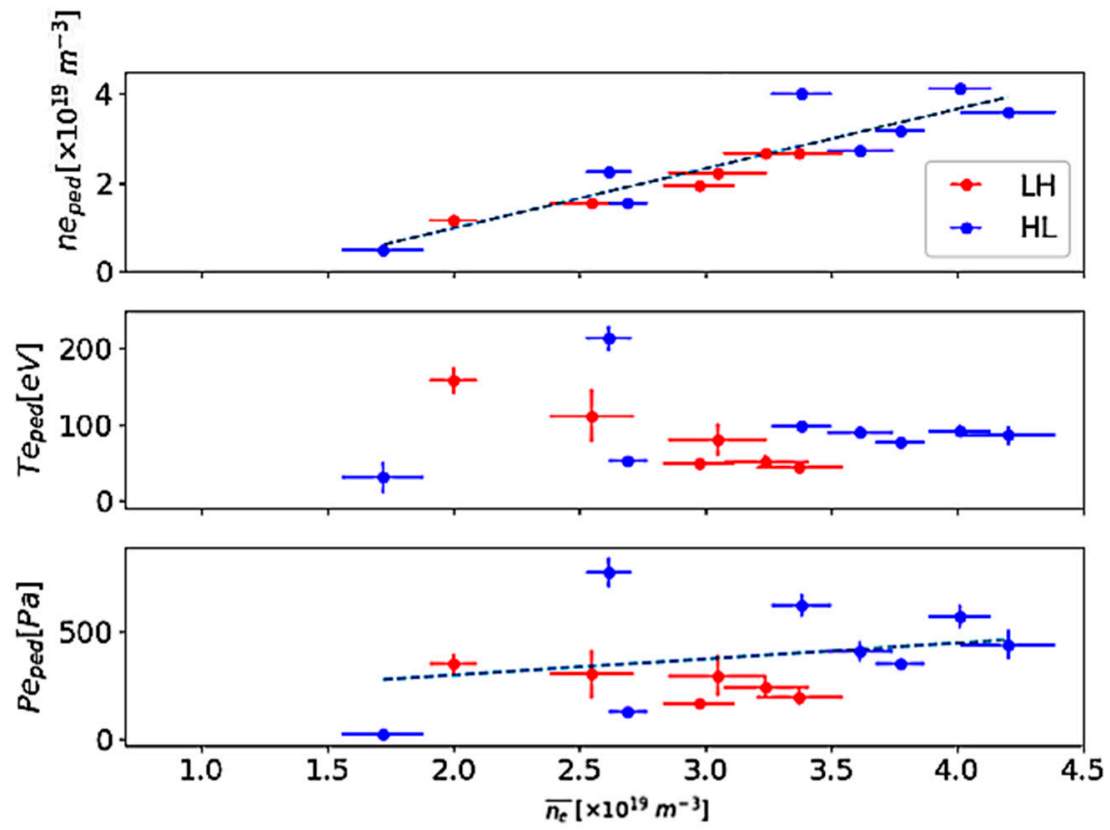

(b)

Figure 4. (a) $P_{t h}$ for the $\mathrm{L}-\mathrm{H}$ and $\mathrm{H}-\mathrm{L}$ transitions and (b) $n_{e}, T_{e}$, and $P_{e}$ at the $n_{e}$ pedestal top or knee, plotted as a function of core $\overline{n_{e}}$.

\section{X-Point Height Dependence}

Data have been analyzed for CDND shots with different X-point heights at $B_{T}=0.53 \mathrm{~T}$. Both the upper and lower $X$-point heights were varied by the same amount over these scans, resulting in associated changes to the magnetic elongation. The lower strike points remained on the horizontal floor of the open MAST divertor for the entire X-point height scan. Hence, the scrape-off layer (SOL) connection length also varied over the $\mathrm{X}$-point height variation. 
The values of $P_{t h}$ at the $\mathrm{L}-\mathrm{H}$ and $\mathrm{H}-\mathrm{L}$ transitions $\left(P_{L H}\right.$ and $\left.P_{H L}\right)$ have been normalized to $\overline{n_{e}}$, and are plotted as a function of lower X-point distance from the divertor floor for the values of $I_{p}=0.67-0.75 \mathrm{MA}, I_{p}=0.75-0.77 \mathrm{MA}$, and $I_{p}=0.77-0.91 \mathrm{MA}$, in Figure 5 . The power threshold for $\mathrm{H}$-mode access and exit increases by a factor of three as the lower X-point distance from the divertor floor increases from $0.38-0.48 \mathrm{~m}$ for $I_{p}=0.67-0.75 \mathrm{MA}$ and from $0.38-0.50 \mathrm{~m}$ for $I_{p}=0.75-0.91 \mathrm{MA}$. The linear dependence of $P_{t h}$ on X-point height disappears for heights above $0.50 \mathrm{~m}$. This is in agreement with earlier results presented from MAST for a smaller X-point height range and limited dataset for single null and double null magnetic configurations [14]. Previous studies on the Joint European Torus (JET) have also shown a similar trend of reduced $P_{\text {th }}$ with lowered $X$-point height was only observed for discharges with the X-point less than $6 \mathrm{~cm}$ from the septum top and the inner and outer strike points and SOL on the horizontal targets [22]. The X-point height dependence of the $P_{t h}$ is also shown to be sensitive to the plasma current, decreasing $I_{p}$ from $0.77 \mathrm{MA}$ to $0.65 \mathrm{MA}$ lower $P_{\text {th }}$ by a factor of three and shifting the $P_{\text {th }}$ roll-over height by $3 \mathrm{~cm}$ to a higher value.

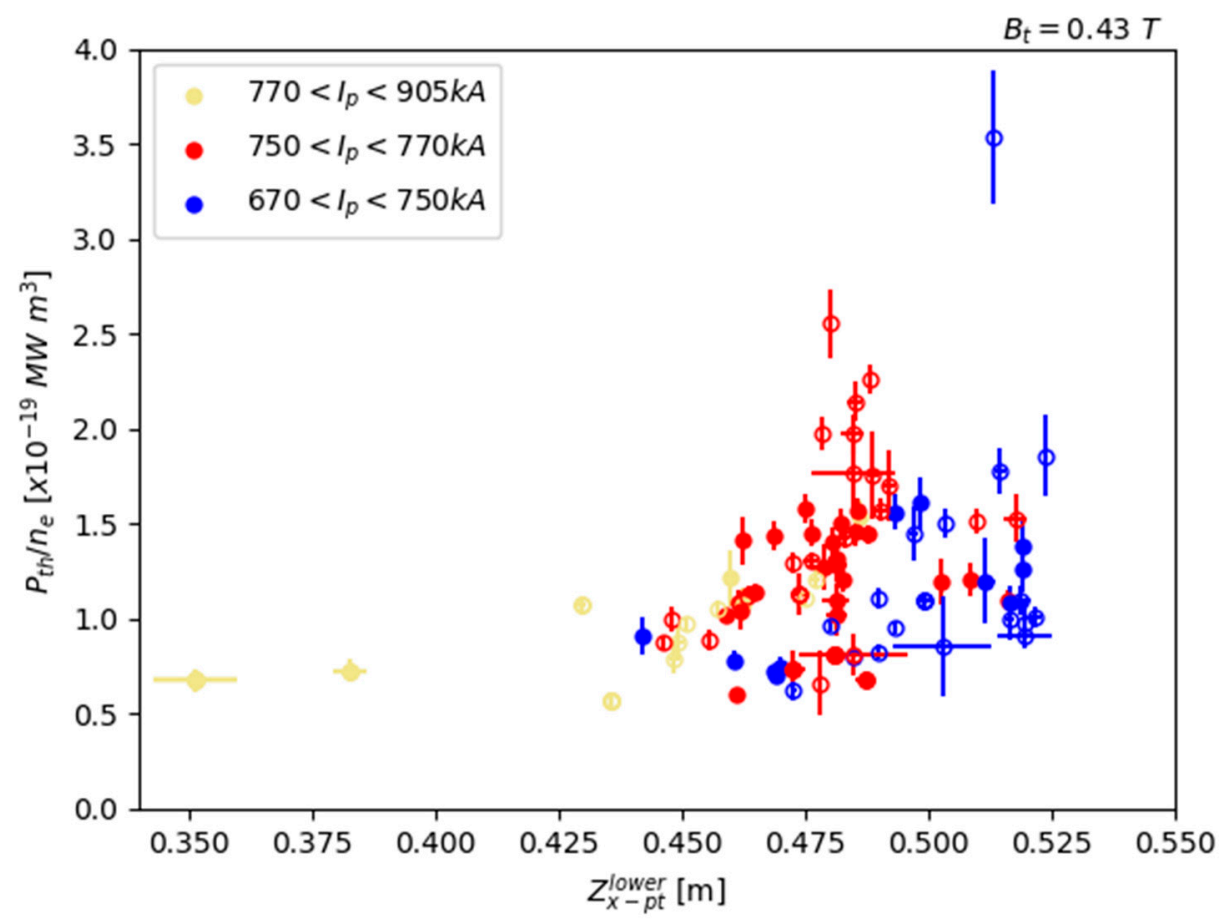

Figure 5. Normalized power threshold for the L-H (closed symbols) and H-L (open symbols), as a function of distance of the lower X-point from the divertor floor for three different values of $I_{p}$.

The increase in $P_{L H}$ and $P_{H L}$ correlates well with decreasing divertor neutral density with increased $\mathrm{X}$-point height. Comparison of the outer divertor $\mathrm{D}_{\alpha}$ intensity in Figure $6 \mathrm{a}, \mathrm{b}$, indicates that the divertor neutral density increases in the vicinity of the $\mathrm{X}$-point with reduced $\mathrm{X}$-point height.

The power dependence of the $\mathrm{L}-\mathrm{H}$ and $\mathrm{H}-\mathrm{L}$ transitions therefore appears to be sensitive to increased vertical proximity between the region of recycling, horizontal target plates, and the X-point. A similar correlation between the $P_{t h}, \mathrm{X}$-point height and divertor $\mathrm{D}_{\alpha}$ intensity was observed on JET [22] and correlated to changes in the sub-divertor neutral pressure by Maggi et al. [23]. The effect of X-point neutral fueling has previously been considered by Toda et al. [24] to explain the experimental observation of H-mode triggering on JFT-2M by gas puffing near the $\mathrm{X}$-point. Toda et al. have shown that for a given set of parameters, there exists a critical value of neutral density near the X-point above which the H-mode bifurcation occurs, due to increased ion losses. More recent simulations by Battaglia et al. [20] have shown that the heat flux through the plasma edge varies strongly with divertor recycling; the relationship between edge $T_{i}$ and the heat flux is dominated by ion-neutral physics, 
which in turn influence $P_{t h}$. It is important to note that MAST had an open divertor, which will have minimized the effect of variation in neutral recycling with X-point height.

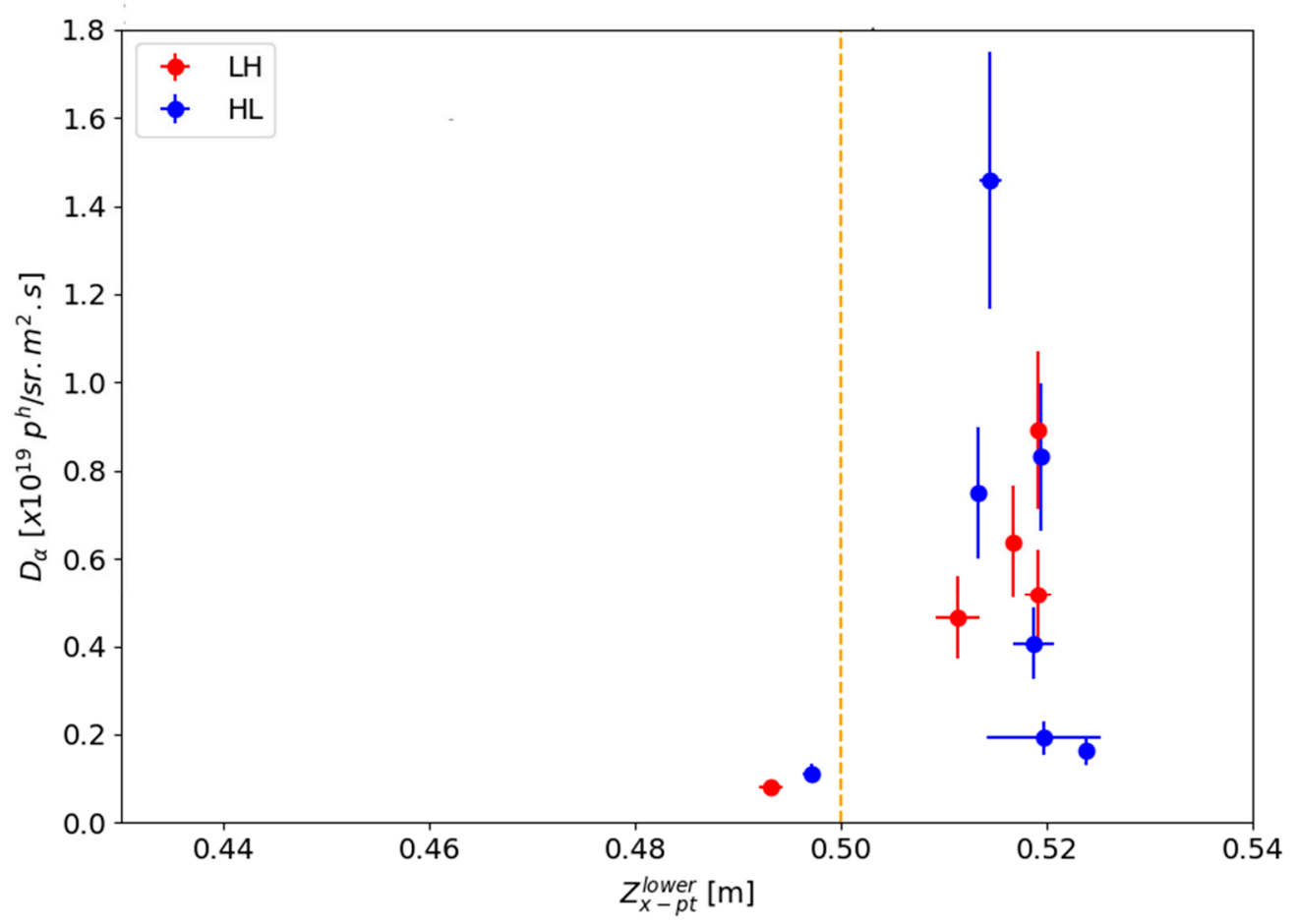

(a)

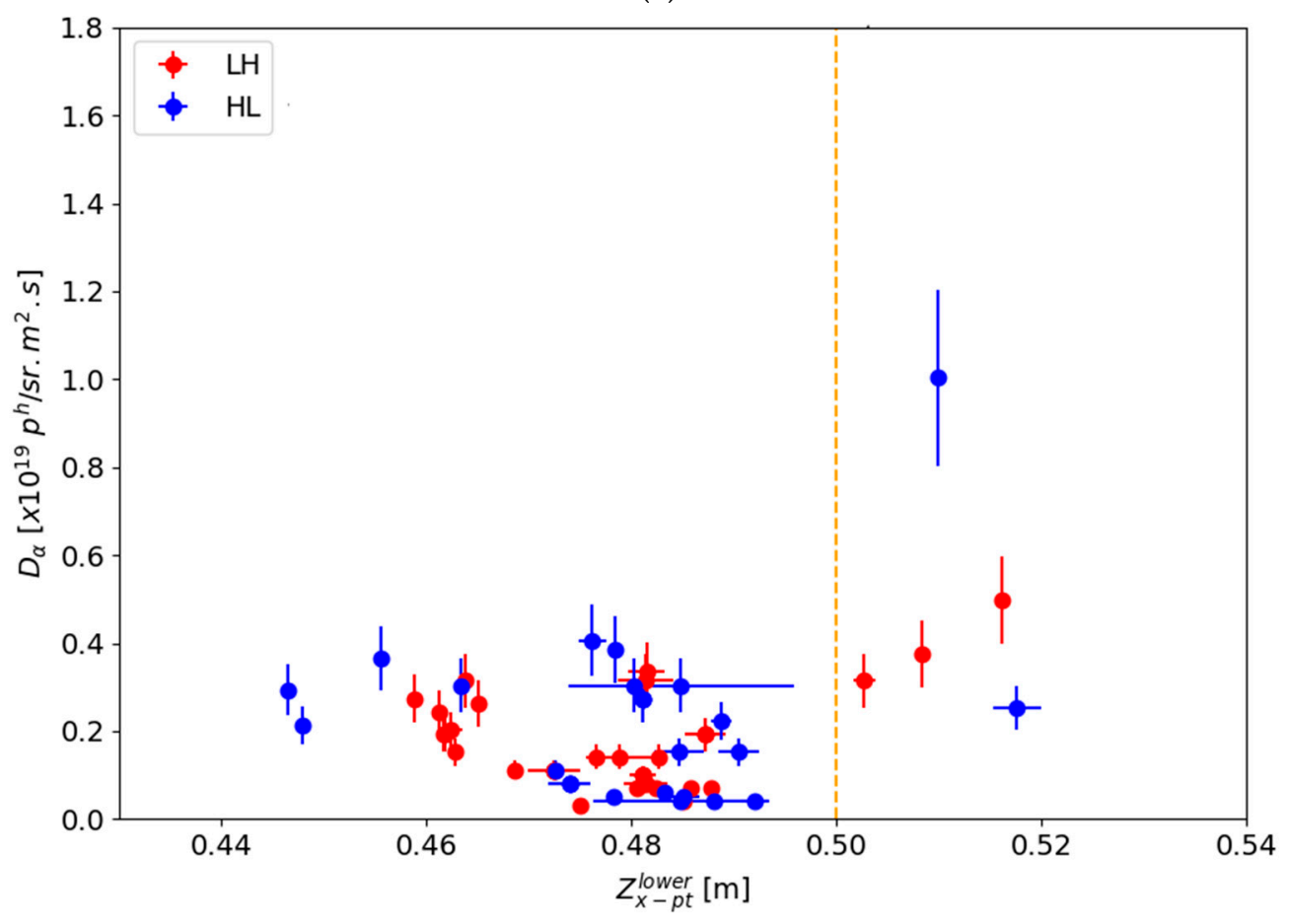

(b)

Figure 6. Divertor $\mathrm{D}_{\alpha}$ intensity at the $\mathrm{L}-\mathrm{H}$ and $\mathrm{H}-\mathrm{L}$ transitions plotted as a function of lower $\mathrm{X}$-point height distance from the divertor floor for (a) $I_{p}=0.67-0.75 \mathrm{MA}$ and (b) $I_{p}=0.75-0.77 \mathrm{MA}$. 


\section{Comparison with Theory}

The MAST data from the density and X-point scans have been compared with the finite beta drift wave model in which edge plasma turbulence is thought to be suppressed through self-generated zonal flows $[9,25]$. Guzdar et al. [9] developed a simple theory for the generation of zonal flows. These investigations indicated the important dimensionless parameter that determines the growth rate of the zonal flow is,

$$
\hat{\beta}=\frac{1}{2} \beta\left(\frac{q R}{L_{n}}\right)^{2}
$$

where, $\beta$ is the ratio of plasma pressure to the magnetic pressure, $q$ is the safety factor, $R$ is the major radius $(\mathrm{m})$, and $L_{n}$ is the density gradient scale length $(\mathrm{m})$. As a function of $\hat{\beta}$, the growth rate for zonal flows has a minimum at $\hat{\beta}_{c}$, which is identified as the threshold point for the $\mathrm{L}-\mathrm{H}$ transition. For $\hat{\beta}>\hat{\beta}_{c}$ the zonal flow stabilization and suppression of fluctuations leads to a steepening of the density gradient and would trigger the transition to H-mode. A simple threshold condition was derived by Guzdar et al. for the $\mathrm{L}-\mathrm{H}$ transitions in tokamaks,

$$
\theta_{c}=0.45 \frac{B_{t}^{2 / 3} Z_{e f f}^{1 / 3}}{\left(R A_{i}\right)^{\frac{1}{6}}}
$$

where $Z_{\text {eff }}$ is the effective plasma ion charge and $A_{i}$ is the ion mass relative to hydrogen. The pedestal parameters $T_{e}$ and $L_{n}$ are the values at the location of the steepest part of the $n_{e}$ pedestal gradient in the edge region of the plasma, just within the last closed flux surface. For a given plasma, the parameter,

$$
\theta=T_{e} /\left(L_{n}\right)^{\frac{1}{2}}
$$

which varies in time, and has to reach the critical value, $\theta_{c}$, to trigger the transition to $\mathrm{H}$-mode according to the model.

A statistical comparison of experiment with theory has been carried out by comparing the observed values of $T_{e}$ at the location of steepest $n_{e}$ gradient for data points in the L-mode and H-mode phases of the shots included in the density and X-point height scans presented in the previous sections. These experimental values of $T_{e}$ are plotted as a function of calculated $T_{e c}$ in Figure 7 for 459 data points, along with the dotted $T_{e}=T_{e c}$ line. The $\mathrm{L}-\mathrm{H}$ transition had been identified for the $P_{t h}$ analysis described earlier, allowing the L- and H-mode states of the plasma to be parameterized. The full set of discharges had scans in $I_{p}=0.67-0.91 \mathrm{MA}, B_{t}=0.43 \mathrm{~T}, n_{e}=1-4.5 \times 10^{19} \mathrm{~m}^{-3}$, and $h_{x p o i n t}=0.35-0.52 \mathrm{~m}$ and have been included in this analysis. As in earlier studies, a value of $Z_{\text {eff }}=2$ has been used [11]. There is a clear separation between the L-mode and H-mode data points across the dotted $T_{e}=T_{e c}$ line, with the $\mathrm{L}-\mathrm{H}$ and $\mathrm{H}-\mathrm{L}$ data points lying either side and fairly close to it. The outlying data show no clear trend, with $11 \%$ of the L-mode points lying above the $T_{e}=T_{e c}$ line and $14 \% \mathrm{H}$-mode points lying below it.

This data demonstrates the finite beta drift wave model provides a reliable onset condition for the transition into and out of H-mode. Even though $P_{t h}$ over the density scan increases by a factor of two and $P_{t h}$ increases by a factor of three over the X-point height scan included in this dataset, the model's dotted line separates the L-mode and H-mode data points well. It is interesting to note that the H-L transition points also occur close to the value of $T_{e c}$, with no evidence of hysteresis in the pedestal parameters. The identifying critical edge parameter for the $\mathrm{L}-\mathrm{H}$ transition is crucial to understanding the physics of the trigger mechanism for bifurcation of the state. This analysis suggests that $T_{e}$ at the location of the steepest $n_{e}$ gradient, along with the evolution of the density gradient scale length (or a related parameter such as $T_{i}$ or $E_{r}$ ), affects the $\mathrm{L}-\mathrm{H}$ and $\mathrm{H}-\mathrm{L}$ transitions. These results also indicate that the pedestal electron density gradient could be one of the contributory parameters controlling H-mode access, supporting the class of theories based on turbulence driven, electron drift waves, which predict the $\mathrm{L}-\mathrm{H}$ transition to occur when $\theta$ reaches a critical value which is 
proportional to $T_{e} /(L n)^{1 / 2}$. It is important to note that the results presented here may provide supporting experimental evidence for the finite beta drift wave model; however, detailed and direct measurements of zonal plasma flow velocities in the pedestal are needed to understand their role in the suppression of turbulence at the $\mathrm{L}-\mathrm{H}$ transition.

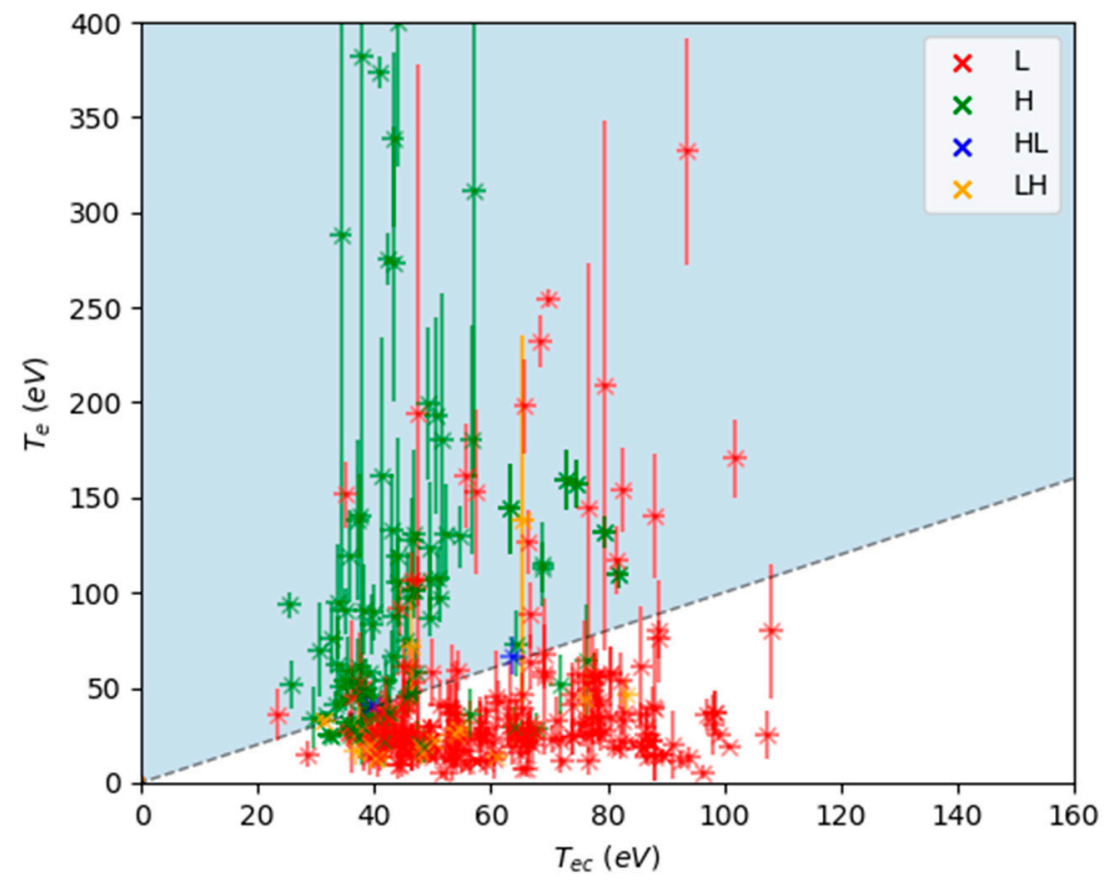

Figure 7. $T_{e}$, taken at the point of steepest edge plasma $n_{e}$ gradient for L-mode, $\mathrm{H}$-mode, $\mathrm{L}-\mathrm{H}$ transitions and $\mathrm{H}-\mathrm{L}$ transitions for shots included in the $\mathrm{n}_{\mathrm{e}}$ and X-point height scans, plotted as a function of $T_{\mathcal{e c}}$ from the finite beta drift wave model. The dotted line represents $T_{e}=T_{e c}$.

\section{Conclusions}

In this paper the power threshold for the $\mathrm{L}-\mathrm{H}$ and $\mathrm{H}-\mathrm{L}$ transitions on MAST have been presented for core averaged density and $\mathrm{X}$-point height scans for CDND magnetic configurations. The $\mathrm{L}-\mathrm{H}$ transition was found to have the dependence, $P_{L H} \propto{\overline{n_{e}}}^{0.77( \pm 0.01)}$. The H-L $P_{\text {th }}$ was found to have a weaker dependence on the averaged core electron density, although the limited amount of data means this indication of power threshold hysteresis at the highest densities and will be an area of planned future experimental investigation on MAST-U.

The strong influence of increasing X-point distance from the divertor floor on raising $P$ th for both the $\mathrm{L}-\mathrm{H}$ and $\mathrm{H}-\mathrm{L}$ transitions, with an increase on $P_{t h}$ by a factor of three over a range of 10-12 cm, is clearly shown. The X-point height dependence of the $P_{\text {th }}$ is also shown to be sensitive to the plasma current; decreasing $\mathrm{I}_{\mathrm{p}}$ from $0.77 \mathrm{MA}$ to $0.65 \mathrm{MA}$ lowers $P_{\text {th }}$ across the $\mathrm{X}$-point height scan by a factor of three and increases the $P_{\text {th }}$ roll-over height by $3 \mathrm{~cm}$. The sensitivity of the L-H and H-L $P_{\text {th }}$ over the relatively specific $X$-point height range, is likely to be related to divertor recycling patterns, proximity of the X-point to neutral source, and ion-neutral interaction in the scrape-off layer, and edge plasma which in turn influences the scrape-off layer and edge plasma $E_{r}$. The role of divertor recycling, ion-neutral interaction, and divertor magnetic geometry in the transitions to and from H-mode will be studied in detail in forthcoming experiments on MAST-U.

Finally, the data included in this study have been compared with the finite beta drift wave model, which provides a reliable onset condition for the transition into and out of H-mode. Even though $P_{\text {th }}$ over the density scan increases by a factor of two and $P_{\text {th }}$ increases by a factor of three over the X-point height scan included in this dataset, the model separates the L-mode and H-mode data 
points reasonably well. The H-L transition points also occur close to the value of $T_{e c}$, with no evidence of hysteresis in the pedestal parameters.

The identifying critical edge parameter for the $\mathrm{L}-\mathrm{H}$ transition remains crucial to understanding the physics of the trigger mechanism for bifurcation of the plasma state. This data suggests that the $T_{e}$ at the location of the steepest $n_{e}$ gradient, along with the evolution of the density gradient scale length (or a related parameter such as $T_{i}$ or $E_{r}$ ), have contributory roles in the $\mathrm{L}-\mathrm{H}$ and $\mathrm{H}-\mathrm{L}$ transitions. The experimental results presented here may provide supporting experimental evidence for the of the finite beta drift wave model for the $\mathrm{L}-\mathrm{H}$ transition. However, direct measurements of zonal plasma flow in the pedestal region, with sufficiently high time and spatial resolution, are required to further understand its role in the suppression of turbulence at the forward and back $\mathrm{H}$-mode transitions.

Author Contributions: Conceptualization, Y.A.; methodology, Y.A.; software, J.-P.B., R.B., T.J.; formal analysis, Y.A., J.-P.B., R.B., T.J.; investigation, Y.A., J.-P.B., R.B., T.J.; writing-original draft preparation, Y.A.; writing-review and editing, Y.A., J.-P.B., R.B., T.J.; supervision; project administration, Y.A.

Funding: These experiments were funded by the RCUK Energy Programme under grant EP/15014045 and the European Communities under the contract of Association between EURATOM and CCFE. The views and opinions expressed herein do not necessarily reflect those of the European Commission.

Acknowledgments: We would like to thank A Kirk and H Meyer for useful discussions and guidance. We would also like to thank the MAST Team for these experiments, data access and collaboration.

Conflicts of Interest: The authors declare no conflict of interest.

\section{References}

1. Wagner, F.; Becker, G.; Behringer, K.; Campbell, D.; Eberhagen, A.; Engelhardt, W.; Fussmann, G.; Gehre, O.; Gernhardt, J.; Gierke, G.V.; et al. Regime of Improved Confinement and High Beta in Neutral Beam Heated Divertor Discharges of the ASDEX Tokamak. Phys. Rev. Lett. 1982, 49, 1408. [CrossRef]

2. Loarte, A.; Huijsmans, G.; Futatani, S.; Baylor, L.R.; Evans, T.E.; Orlov, D.M.; Schmitz, O.; Becoulet, M.; Cahyna, P.; Gribov, Y.; et al. Progress on the Application of ELM Control Schemes to ITER Scenarios from the Non-Active Phase to DT Operation. Nucl. Fusion 2014, 54, 033007. [CrossRef]

3. Lazarus, E.A.; Navratil, G.A.; Greenfield, C.M.; Strait, E.J.; Austin, M.E.; Burrell, K.H.; Casper, T.A.; Baker, D.R.; DeBoo, J.C.; Doyle, E.J.; et al. Higher Fusion Power Gain with Current and Pressure Profile Control in Strongly Shaped DIII-D Tokamak Plasmas. Phys. Rev. Lett. 1996, 77, 2714. [CrossRef] [PubMed]

4. Sabbagh, S.A.; Fredrickson, E.D.; Mansfield, D.K.; Bell, M.G.; Batha, S.H.; Bell, R.E.; Budny, R.V.; Bush, C.E.; Chang, Z.; Hawryluk, R.J.; et al. Deuterium-Tritium TFTR Plasmas with High Internal Inductance. In Proceedings of the 16th International Conference on Fusion Energy, Montreal, QC, Canada, 7-11 October 1996; Volume 1, p. 921.

5. Peeters, A.G. The Bootstrap Current and its Consequences. Plasma Phys. Control. Fusion 2000, 42, B231-B242. [CrossRef]

6. Martin, Y.R.; Takizuka, T. ITPA CDBM H-mode Threshold Database Working Group. Power Requirement for Accessing the H-mode in ITER. J. Phys. Conf. Ser. 2008, 123, 012033. [CrossRef]

7. Takizuka, T. Roles of aspect ratio, absolute $\mathrm{B}$ and effective $\mathrm{Z}$ of the H-mode power threshold in tokamaks of the ITPA database. Plasma Phys. Control. Fusion 2004, 46.5A, A227. [CrossRef]

8. Campbell, D.J. The ITER Research Plan. In Proceedings of the 24th International Conference on Fusion Energy, San Diego, CA, USA, 8-13 October 2012.

9. Guzdar, P.N.; Kleva, R.G.; Das, A.; Kaw, P.K. Zonal Flow and Zonal Magnetic Field Generation by Finite Beta Drift Waves: A Theory for Low to High Transitions in Tokamaks. Phys. Rev. Lett. 2001, 87, 015001. [CrossRef] [PubMed]

10. Counsel, G.F.; Ahn, J.-W.; Akers, R.; Arends, E.; Buttery, R.; Field, A.R.; Gryaznevich, M.; Helander, P.; Kirk, A.; Meyer, H. Confinement and Exhaust in the Mega Ampere Spherical Tokamak. Plasma Phys. Control. Fusion 2002, 44, B23. [CrossRef]

11. Meyer, H.; Carolan, P.G.; Conway, G.D.; Cunningham, G.; Horton, L.D.; Kirk, A.; Maingi, R.; Ryter, F.; Saarelma, S.; Schirmer, J.; et al. The MAST, ASDEX Upgrade and NSTX teams. H-mode Physics of Near 
Double Null Plasmas in MAST and its Application to other Tokamaks. Nucl. Fusion 2006, 46, 64-72. [CrossRef]

12. Meyer, H.; Akers, R.J.; Alladio, F.; Appel, L.C.; Axon, K.B.; Ben Ayed, A.; Boerner, P.; Buttery, R.J.; Carolan, P.G.; Ciric, D.; et al. Overview of Physics Results from MAST. Nucl. Fusion 2009, 49, 104017. [CrossRef]

13. Kirk, A.; O'Gorman, T.; Saarelma, S.; Scannell, R.; Wilson, H. The MAST team. A Comparison of H-mode Pedestal Characteristics in MAST as a Function of Magnetic Configuration and ELM Type. Plasma Phys. Control. Fusion 2009, 51, 065016. [CrossRef]

14. Meyer, H.; De Bock, M.F.M.; Conway, N.J.; Freethy, S.J.; Gibson, K.; Hiratsuka, J.; Kirk, A.; Michael, C.A.; Morgan, T.; Scannell, R.; et al. L-H Transition and Pedestal Studies on MAST. Nucl. Fusion 2011, 51, 113011. [CrossRef]

15. Scannell, R.; Walsh, M.J.; Carolan, P.G.; Conway, N.J.; Darke, A.C.; Dunstan, M.R.; Hare, D.; Prunty, S.L. Enhanced Edge Thomson Scattering on MAST. Rev. Sci. Instrum. 2006, 77, 10E510. [CrossRef]

16. Gibson, K.J.; Barratt, N.; Chapman, I.; Conway, N.; Dunstan, M.R.; Field, A.R.; Garzotti, L.; Kirk, A.; Lloyd, B.; Meyer, H.; et al. New Physics Capabilities from the Upgraded Thomson Scattering Diagnostic on MAST. Plasma Phys. Control. Fusion 2010, 52, 124041. [CrossRef]

17. Bähner, J.P. L-H Transition and Pedestal Studies in MAST. Master's Thesis, Imperial College London, London, UK, 2018.

18. Andrew, Y.; Hawkes, N.C.; O’Mullane, M.G.; Sartori, R.; Beurskens, M.N.A.; Coffey, I.; Joffrin, E.; Loarte, A.; McDonald, D.C.; Prentice, R.; et al. Edge Ion Parameters at the L-H Transition on JET. Plasma Phys. Control. Fusion 2004, 46, 337. [CrossRef]

19. Sauter, P.; Putterich, T.; Ryter, F.; Viezzer, E.; Wolfrum, E.; Conway, G.D.; Fischer, R.; Kurzan, B.; McDermott, R.M.; Rathgeber, S.K. L- to H-mode Transitions at Low Density in ASDEX Upgrade. Nucl. Fusion 2012, 52, 012001. [CrossRef]

20. Battaglia, D.J.; Chang, C.S.; Kaye, S.M.; Kim, K.; Ku, S.; Maingi, R.; Bell, R.E.; Diallo, A.; Gerhardt, S.; LeBlanc, B.P.; et al. The NSTX Team. Dependence of the L-H transition on X-point geometry and divertor recycling on NSTX. Nucl. Fusion 2013, 53, 113032. [CrossRef]

21. Andrew, Y.; Sartori, R.; Righi, E.; de la Luna, E.; Hacquin, S.; Howell, D.; Hawkes, N.C.; Horton, L.D.; Huber, A.; Korotkov, A.; et al. JET-EFDA contributors. H-mode Access in the Low Density Regime on JET. Plasma Phys. Control. Fusion 2006, 48, 479. [CrossRef]

22. Andrew, Y.; Hawkes, N.C.; O’Mullane, M.G.; Sartori, R.; de Baar, M.; Coffey, I.; Guenther, K.; Jenkins, I.; Korotkov, A.; Lomas, P.; et al. JET-EFDA Contributors. JET Divertor Geometry and Plasma Shape Effects on the L-H Transition Threshold. Plasma Phys. Control. Fusion 2004, 46, A87-A93. [CrossRef]

23. Maggi, C.F.; Delabie, E.; Biewer, T.M.; Groth, M.; Hawkes, N.C.; Lehnen, M.; de la Luna, E.; McCormick, K.; Reux, C.; Rimini, F. L-H Power Threshold Studies in JET with Be/W and C wall. Nucl. Fusion 2014, 54, 023007. [CrossRef]

24. Toda, S.; Itoh, S.-I.; Yagi, M.; Miura, Y. A Theortical Model of H-mode Transition Triggered by Condensed Neutrals near X-point. Plasma Phys. Control. Fusion 1997, 39, 201-312. [CrossRef]

25. Guzdar, P.N.; Kleva, R.G.; Groebner, R.J.; Gohil, P. Low to High Confinement Transition Theory of Finite Beta Drift-Wave Driven Shear Flow and its Comparison with Data from DIII-D. Phys. Plasmas 2004, 11, 1109. [CrossRef]

(C) 2019 by the authors. Licensee MDPI, Basel, Switzerland. This article is an open access article distributed under the terms and conditions of the Creative Commons Attribution (CC BY) license (http://creativecommons.org/licenses/by/4.0/). 